\title{
The Interdependence of Humanistic Values and the Level of Existential Fulfillment of an Intellectual Leader
}

\author{
Marianna V. Mikhaylova ${ }^{1, *}$ and Viktoria A. Dmitrieva² \\ ${ }^{1}$ Moscow State Technological University "STANKIN", Department of philosophy end and social management, RU-127055, Moscow, \\ Russia \\ ${ }^{2}$ Russian State Social University, Department of social ontopsychology, RU-129226, Moscow, Russia
}

\begin{abstract}
Value orientations of the young intellectual leaders cause absolute interest because, by realizing their ambitions, projects and making creative breakthroughs, they will eventually change history, create new directions for the development of science and art. Not only the realization of the talented youth's own potential depends on what they think about the meaning of a person, on the worldview positions, but also whether the future society will be humanistic, whether talent will be cultivated in it. The article presents the results of a statistical sociological study of ideas about humanism, qualities of a leader, value orientations and the level of existential fulfillment of young intellectual leaders - graduate students of a technical university. The level of existential fulfillment of the sample is interconnected with a high vitality and selfawareness of health, ideas about humanism as the flourishing of sciences and art, ideas about freedom as a responsible choice, and the morality of universal justice. However, graduate students deliberately downplay the values "stimulation" and "hedonism" (striving for novelty and deep experience to maintain an optimal level of activity). Although the combination of these values with the value "independence" is significant for creative activity.
\end{abstract}

\section{Introduction}

The realization of humanistic values has been in the focus of thinkers in different eras. The main ideas of classical humanism began to be formulated since Socrates, they can be designated as follows: a person has his own internal apriority order, which serves as his criterion of rationality; a person is able to do good and is responsible for his own creative potential and the development of mankind; freedom and human dignity are fundamental values of society [1]. It must be said that the values of humanism are acquiring special significance right now in the period of digital technologies and the increasingly active use of artificial intelligence. The human mind created artificial intelligence, but the idea of a person from the standpoint of existential meaning, longing for one's own being, raises many more questions among talented young people than an ultra-modern "smart" computer. More and more young people admit that social networks, Instagram creates the illusion of pseudo-realization, a race for false values, lead to a decrease in their own selfesteem and an increase in an inferiority complex when it is necessary to compare oneself with a "picture" that is a partial, selective or embellished reflection of reality [2]. This forces the most creative youth, those who can become intellectual leaders, to clarify their own values, cleared of myths and advertising labels in real life. A person capable of developing their potential to the level of functionality for society needs special pedagogy and other life values. "Leaders are generated by vital spontaneity and individual, voluntary, existentialhistorical self-improvement. These people become great and, perhaps, even the best for the sole purpose - the highest service to humanity." [3] Along with the problems of environmental disasters, the search for new types of energy, there is an acute problem of personality, namely the inability of a person to understand his potential, to achieve authenticity (the way of thinking and actions corresponding to his inner uniqueness and innate talent) and consciously apply his mind and abilities in a specific environment with a creative vector. "The human mind is the first wealth, and one of the highest satisfactions is to become the value quotient, the producer of the greatest being, which, thanks to its own realization, also develops the still necessary humanistic civilization." [4] Regardless of the activity field: mathematics or poetry, an intellectual leader also becomes a conductor of social and humanistic values for his environment and those to whom the results of his work are intended. [5] Humanistic ideas awaken a person's awareness of his central place and value in the universe and history [6]. For the first time, the concept of existential fulfillment, as a description of a person's life quality, began to be used by Viktor Frankl. Reflections on the meaning of human life undoubtedly led to the concept of happiness, which is close to hedonistic in philosophy today in the ordinary sense today. Hedonistic 
happiness (Epicurus) is the absence of suffering, wellbeing, fullness of desires, the taste of life. Existential fulfillment is closer to the concept of eudemonic happiness acquired through dignity according to Aristotle [9]. It means the meaningfulness of one's own life, the ability to contribute to life; the value of morality is assessed in direct proportion to happiness; to wish others' happiness is not so much a moral duty as the happiness of others is a condition of one's own happiness.

For a young intellectual leader, his potential determines the way to achieve existential fulfillment. The inability or frivolous attitude towards the realization of one's own natural potential gives a rise to the feeling of existential melancholy, helplessness, guilt, fear, doubt.

\section{Analytical part}

In order to study the interdependence of ideas about the humanistic values of an individual and the level of existential fulfillment of a young intellectual leader, a study was carried out in a sample of graduate students of the Russian higher technical school. The number of participants is 58 . The average age is 25 years. $72 \%$ are male graduate students, $28 \%$ are female graduate students. The author's questionnaire, the questionnaire of moral and value choices, the value questionnaire of Schwartz S. [7], the scale of existence of A. Langle and K. Orgler [8] were used as methods. Frequency, correlation and content analysis were used to study the answers, and an ontopsychological approach was used to interpret the results.

The author's questionnaire made it possible to get an idea of how the position of humanism is perceived by graduate students. What do graduate students mean by the concept of humanism. What qualities are considered the most important for the realization of human potential.

\subsection{Humanism, humanistic society}

The concept of humanism for graduate students is: "spiritual health" and "decent social support for vulnerable groups of the population" (55.17\%); "The flourishing of arts and science" (27.59\%), "medicine will work so well that there will be no sick people left" (20.69\%), "there will be no illiterate people” (see Fig. 1).

Correlation analysis showed that the opinion "humanism is spiritual health is interconnected (p-0.01) with a high self-esteem of vitality. The opinion "humanism is the flourishing of sciences and art" is interconnected (p-0.05) with the ability to feel the existential significance of an action, to orient itself in actions towards meaning, and not just towards the goal (ST, Längle).

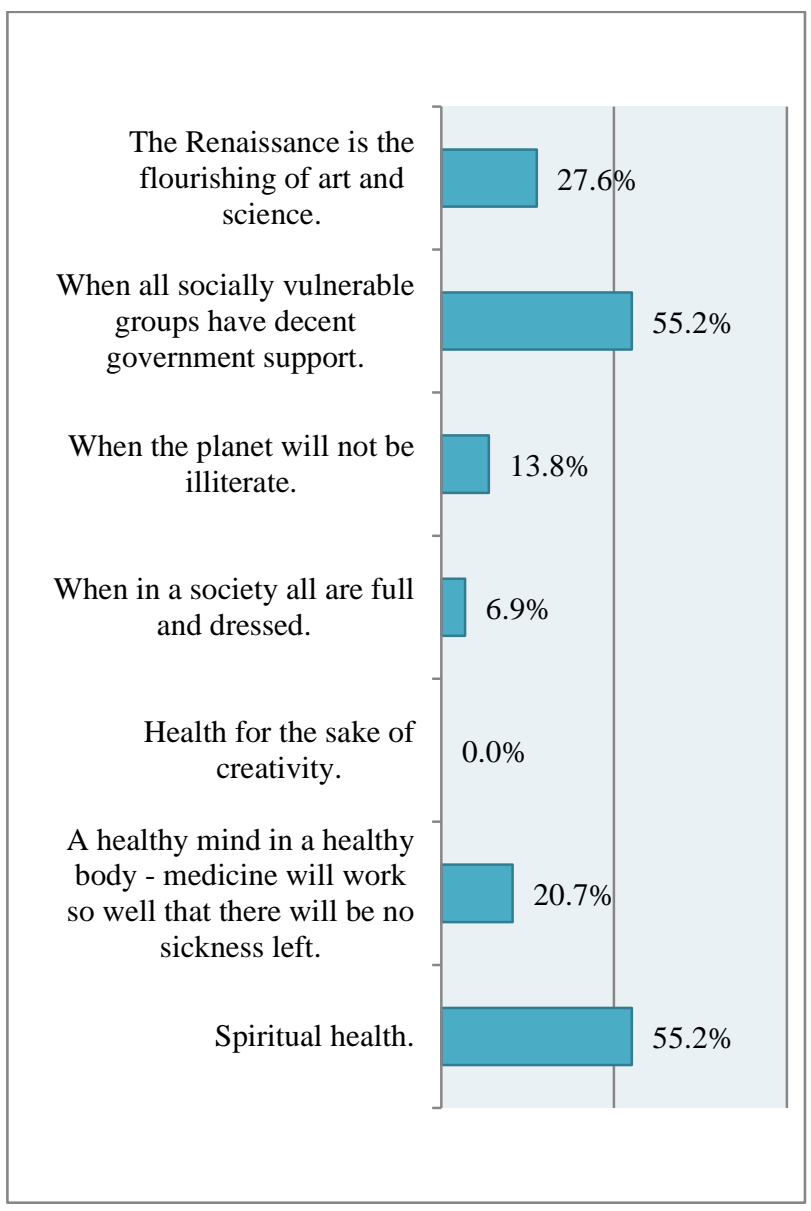

Fig. 1. Associations of graduate students with the concept of humanism.

The question "if we imagine the possibility of the existence of a society consisting of self-realized individuals and geniuses, then what characteristics it would differ" allowed us to determine how much the respondents accept the idea of a society in which everyone has the right and obligation to reveal their talent (see Fig. 3).

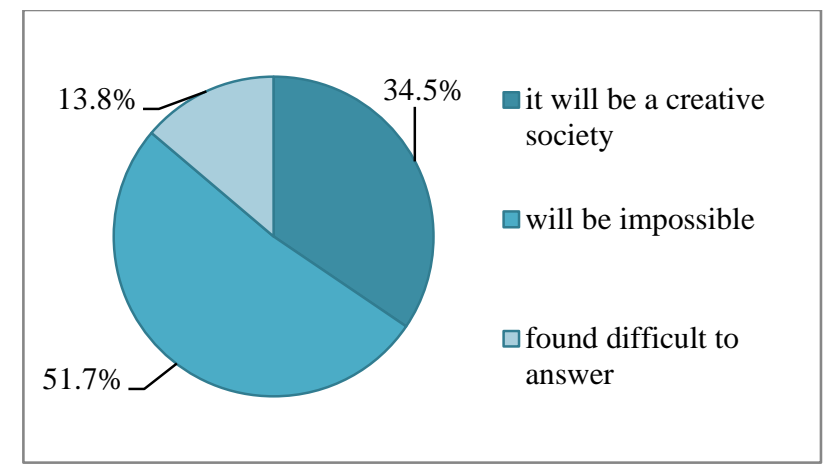

Fig. 2. The opinion of the respondents about the possibility of the existence of a humanistic society.

Almost half of the respondents $51.72 \%$ believe such an idea is impossible, $13.79 \%$ found it difficult to answer and only $34.48 \%$ answered that this is possible, agreeing that such a society would be characterized by creativity, flourishing of culture and various fields of activity. 


\subsection{Self-realization goals}

Ideas about the goals of a person's self-realization reveal the personal orientation of graduate students. Most of $41.38 \%$ consider the goal of self-realization to be "achieving individual happiness"; 37.93\% "disclosure of one's own abilities, potential" is a focus on selfrealization of uniqueness in the classical humanistic sense; $10.34 \%$ "leave a trace in history" - an orientation toward power; none of the respondents chose to "plant a tree, build a house, have children" - a generally accepted stereotype.

Correlation analysis revealed that the goal of selfrealization "disclosing one's own abilities and potential" is interconnected with the leader's quality "love for one's own business" (p-0.01). This relationship is important for the success of scientific activities. There is also a relationship with the opinion that in "a humanistic society, a person is sure that he is the main and responsible actor capable of self-realization," which means a willingness to go his own non-standard way. The goal of "achieving individual happiness" is significantly (p-0.01) interconnected with the idea that a person is a grain of sand in time. The goal "to leave a mark on history" is significantly interconnected (p-0.01) with the value of power (Schwartz) in practice.

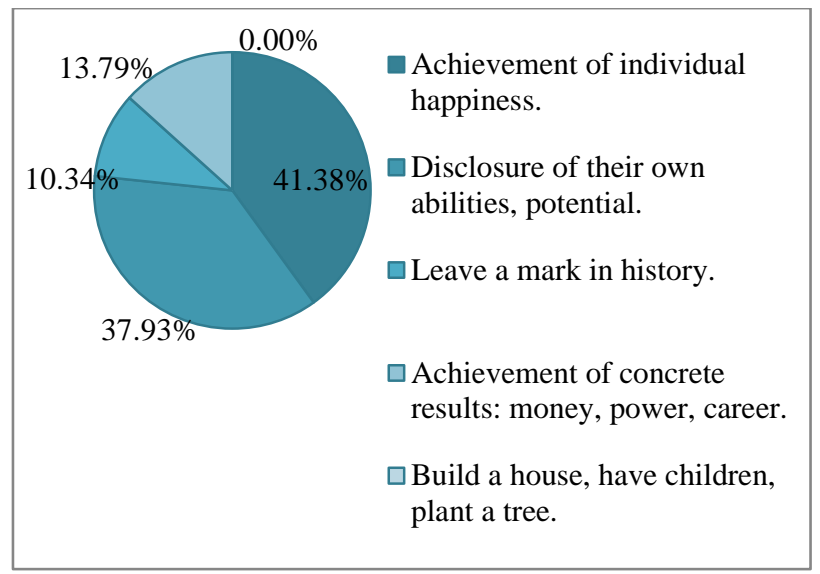

Fig. 3. The goal of human self-realization through the eyes of graduate students.

\subsection{Qualities that cause respect for a Human}

When asked which person evokes the greatest inner respect, the majority (62.07\%) of the respondents answered that this is "a person who fights, acts, is able to transform any problem into victory". About a quarter of the respondents $(24.14 \%)$ believed that the one who is happy and does not depend on anyone else deserves respect. $10.34 \%$ believe that respect should be given to a person who sacrificed his own happiness for the happiness of others, and $3.45 \%$ choose the criterion of respect - achieving high results.

One of the open questions concerned the search for a quality that inspires pride in all of humanity (see Fig. 4). The content analysis of the answers allowed all the answers to be divided into seven blocks: kindness-mercy (28.57\%), purposefulness-responsibility (17.86\%), creativity (14.29\%), responsiveness (7.69\%), intelligence (10.71\%), self-sacrifice $\quad(7.14 \%), \quad$ communication (7.14\%).

The quality of creativity highlighted by the respondents (see Fig. 5) turned out to be significantly (p0.01 ) correlated with high self-esteem of vitality and energy, with the concept of "humanism is spiritual health" and morality "internal duty - responsibility to oneself" - in other words: respondents who consider creativity to be the highest achievement of a person in a society of spiritually healthy people make decisions based on the inner truth of things, an inner ethical principle.

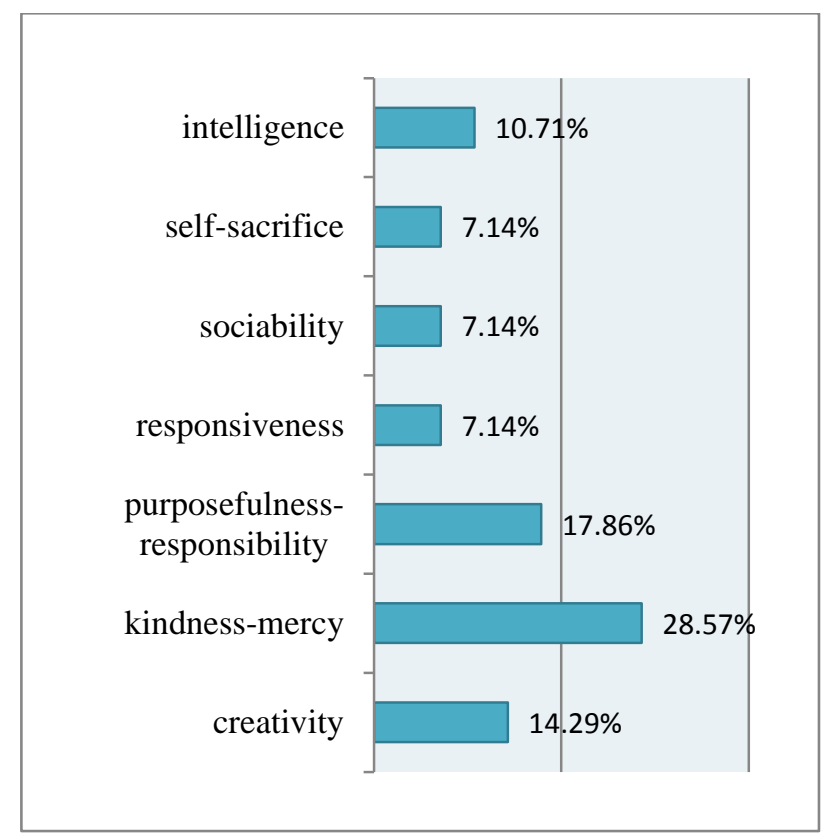

Fig. 4. According to the respondents, the most valuable human quality that inspires pride in humanity.

The opinion that pride in all of humanity evokes human intelligence is interconnected with the idea that in a humanistic society a person is sure that he is the main protagonist (p-0.01), capable of self-realization in a historical context, values of independence and achievement (Schwartz) and is inversely interconnected with the 6th factor of moral choice "role behavior (class ethics in sociology) - in other words, they rely on their intelligence and autonomy to achieve what they want. The opinion that pride in all of humanity causes kindness-mercy is inversely correlated with the 15th factor of moral choice "duty to oneself", with the values of self-achievement (Schwartz) and free emotionality (ST, Längle) - negative correlations seem to indicate ambiguous connotations the concept of "kindness" of the respondents. 


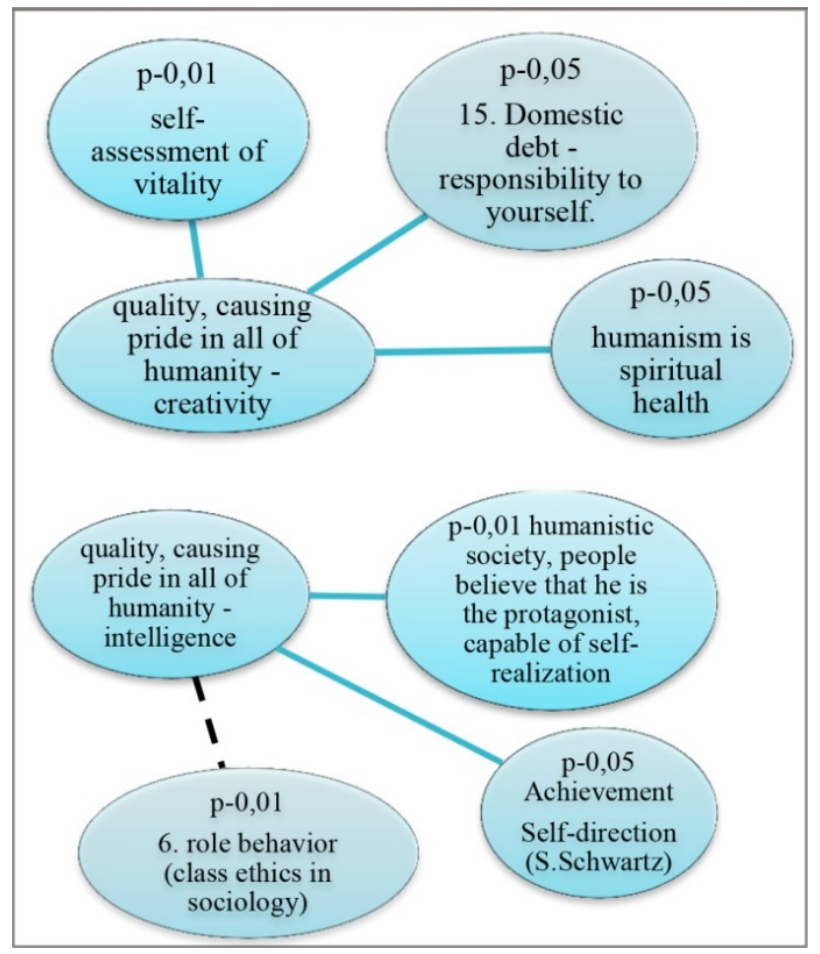

Fig. 5. Correlations with the opinion "creativity causes pride in humanity".

As part of the study of the humanistic orientation of young intellectual leaders, it was interesting to find out what leadership qualities graduate students consider a necessary condition for success. For the scale, a classification of the ontopsychological school was proposed, consisting of seven items, and six more items from the general classification of Stogdill Ralph were added, in total it was proposed to select up to 7 qualities (see Fig. 6). The most important qualities that distinguish an intellectual leader, graduate students consider: "ambition - a strong-willed desire for action", "the highest level of professional knowledge" and "love of one's own business."

The least important characteristics of a leader are recognized as follows: a leader is the expectations of followers (10.34\%), a leader is the result of a special atmosphere, respect for family traditions and technical and rational development consistent with one's own identity. The latter quality, apparently, seems insignificant to graduate students, since the level of their education in the direction chosen more than 6-10 years ago seems to be no longer discussed. However, data analysis suggests a significant relationship between the quality of "intuitive rationality" with indicators of existential fulfillment (Längle). Intuition and rational analysis of intuition is one of the least studied areas of psychology, even though intuition always accompanies creative activity, the creation of new fundamental knowledge and invention. But for our sample, this is also a sensitive quality because engineering is an activity focused on accuracy, since it is associated with the safety of technology and special requirements are imposed on it, and intuition is an irrational way of knowing.

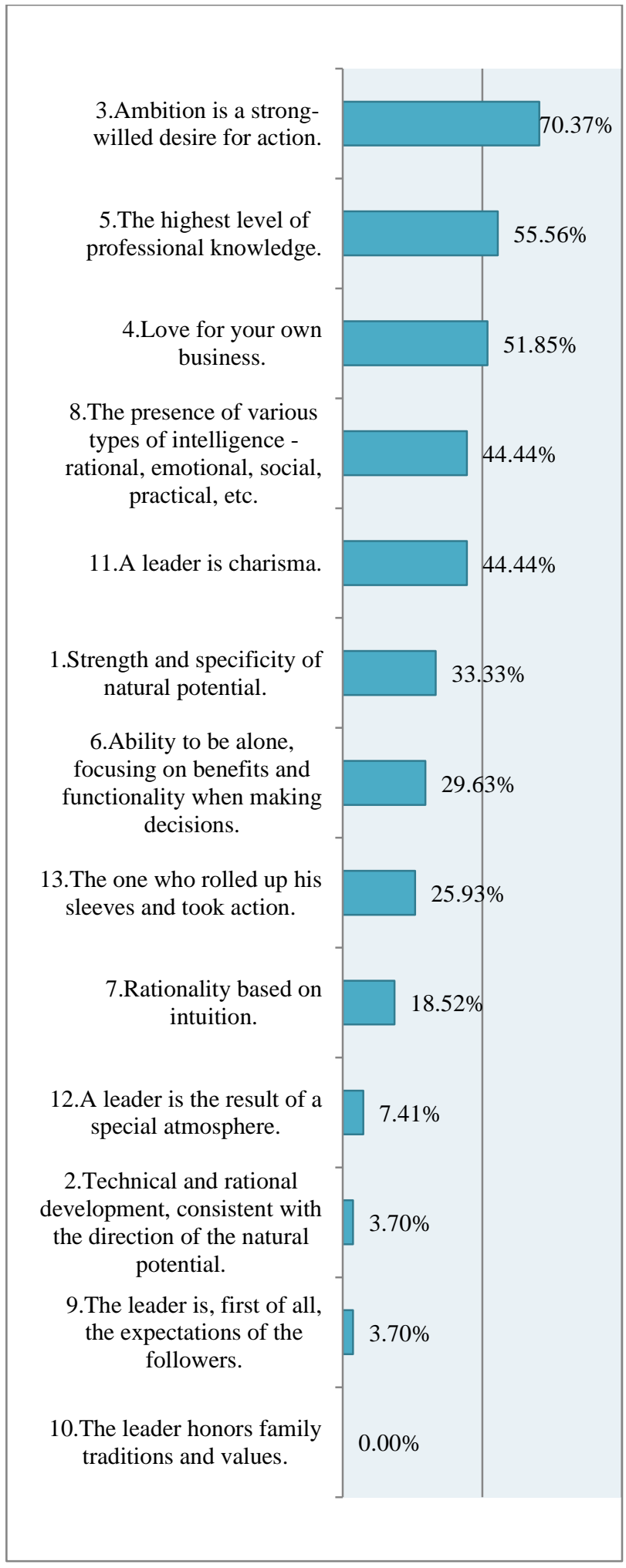

Fig. 6. The qualities necessary for an intellectual leader according to the respondents.

\subsection{Freedom}

"Freedom of choice or free will": $44.83 \%$ of graduate students tend to view freedom as an opportunity of creative development, this opinion is a conscious motivation for self-realization (see Fig. 7). However, this opinion is highly significantly interconnected (p-0.001) 
with the opinion that in a truly humanistic society a person will always receive support and is significantly interconnected (p-0.01) with the factor of moral choice religiosity. $41.38 \%$ of graduate students see freedom as the ability to take responsibility. The opinion that freedom is a responsibility is inversely related (p-0.01) with the value of achieving socially desirable results in life and the value of power (Schwartz) in the personality profile (see Fig. 8). There is a relationship with ST (realism of perception) (p-0.05) and a significant relationship with P-openness of perception of the world and oneself (Längle). That is, graduate students who believe that to be or not to be free is everyone's personal choice, are satisfied with the quality of their lives and are not inclined to achieve socially desirable values. $24.14 \%$ of respondents answered that freedom is a utopia, these graduate students highly appreciate openness to new experience at the level of individual priorities.

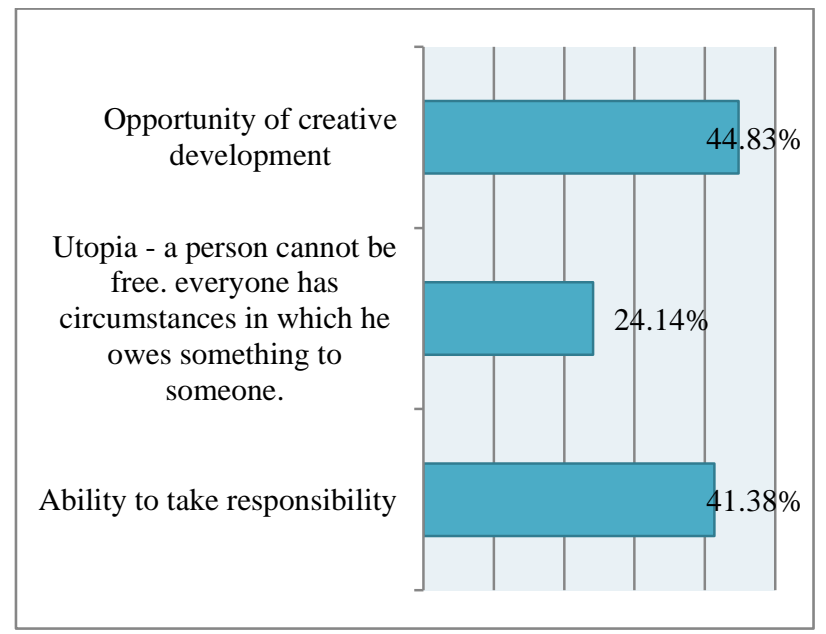

Fig. 7. The respondents' perceptions of freedom.

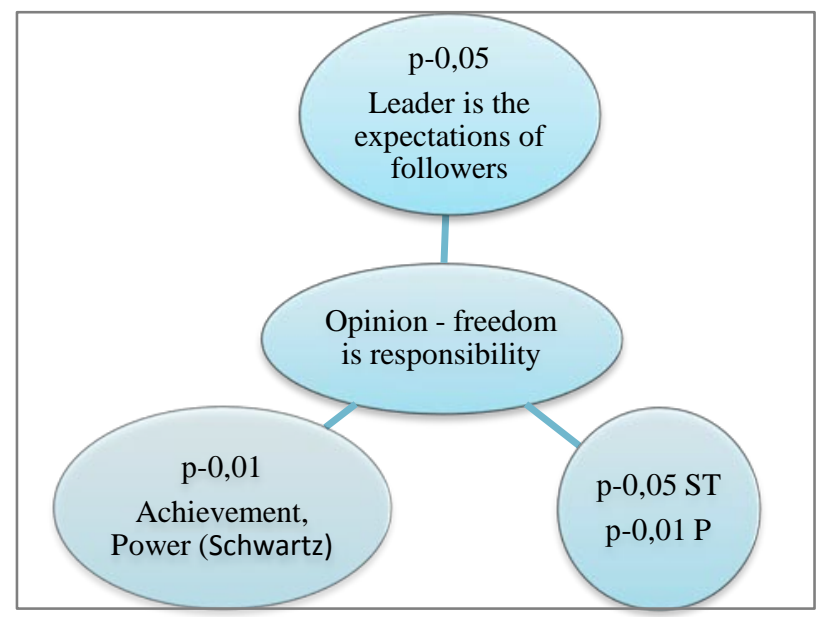

Fig. 8. Interconnection of respondents' ideas about freedom.

\subsection{Analysis of the moral and value choices of graduate students.}

The 16-factor structure of Dubov [10] is a description of the everyday moral consciousness (at least, the moral consciousness of Russians). And even though the principles representing these factors exist in the minds of each individual in the form of completely individual hierarchies, the named 16 meanings are, in their totality, the main system-forming vectors of public morality characteristic of Russian culture. Relying on them, people think about morality, according to the criteria they have designated, they evaluate their own and other people's actions. For a sample of graduate students of a technical university, the following factors of the methodology used are of the greatest importance: 8th factor - "global survival" (this principle can be interpreted as social Darwinism on a global scale) (4.03); 7th factor "hedonism" (it is one of the variants of hedonism in naturalistic schools, for example, in the history of ethics - Democritus, Socrates, it is based on the principle of pleasure that does not depend on the path of its achievement, the desire for this pleasure may contradict accepted moral concepts) (4.00); The second factor "aversion to immorality" is also called "the intrinsic value of morality" (the main motive of this intrinsic value also includes an aesthetic assessment of the situation, while it should be noted that "aversion to immorality" is expressed in absolute terms stronger than the feeling that good is beautiful ) (3.93); 6th factor "role behavior" (class ethics of sociological schools (3.90); 15th - as "internal duty" or "duty for moral behavior to oneself" (3.86) (see Fig. 9).

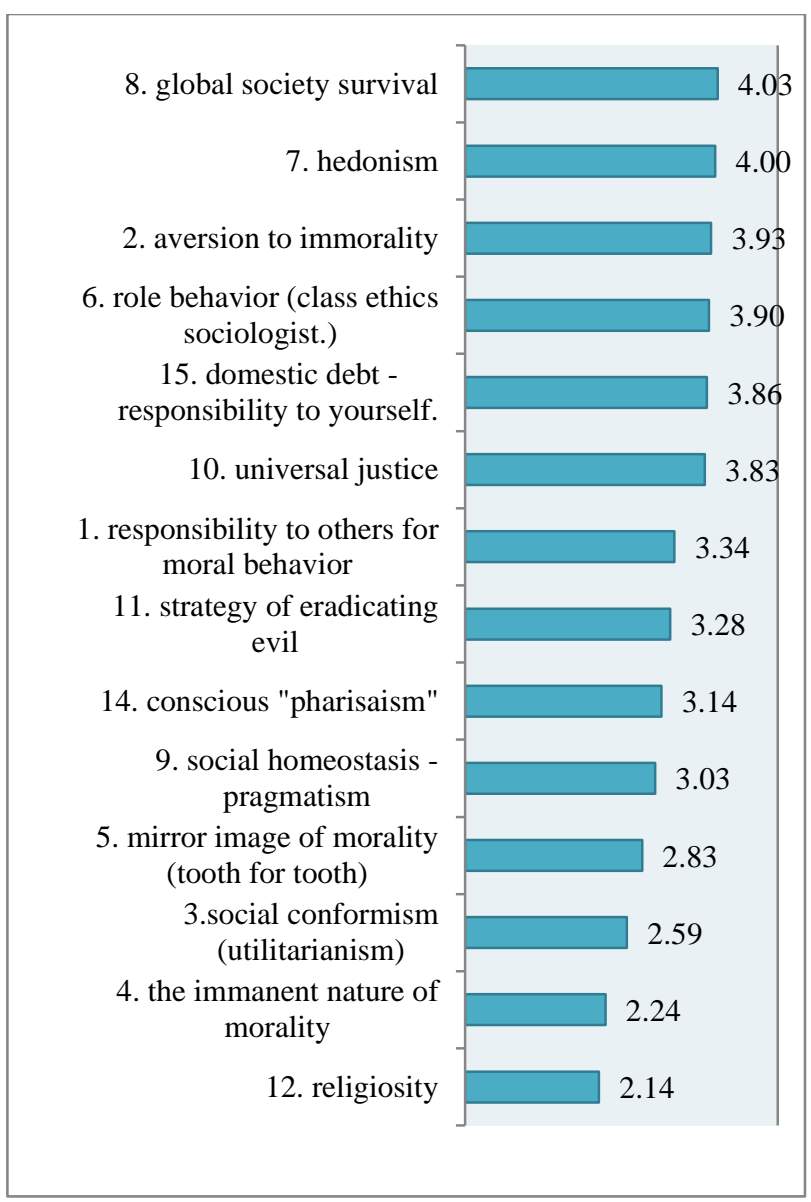

Fig. 9. Results according to the methodology of moral value choices.

According to the research of the author of the methodology (Dubov), the strengthening of 7,8,15 
factors is characteristic of the social feelings of optimists (whose attitude to life can be defined as "everything is going well, and when not very well, I successfully overcome difficulties”). The moral motives of the group of intellectually successful young people we are studying can be designated as heterogeneous, multiple: the interests of society, personal pleasure, striving for moral aesthetics and duty to oneself. Moreover, the 2nd factor "the intrinsic value of morality" is the most important difference between our group and the results described by the author of the methodology in other groups.

\subsection{Analysis and interpretation of the value questionnaire (Schwartz S.)}

The test is usually used to study the dynamics of the transformation of values both in social groups (cultures) and for an individual in connection with his life problems. Values in this questionnaire mean "learned" needs that depend on the culture, environment, mentality of a particular society. The level of normative ideals reflect the conscious beliefs about how to behave. The level of individual priorities diagnoses the values that the subject is guided by in specific situations of his life. The distribution of the respondents' answers showed that the values of universalism (understanding, tolerance and protection of the well-being of all people and nature), independence (independence of thought and choice of methods of action, in creativity and research activity) and security (safety and stability of society, relations and itself yourself). The lowest scores are for hedonism and stimulation (striving for novelty and deep experiences to maintain an optimal level of activity). To construct Schwartz's circle of values, the obtained mean values on the scales were converted into ranks (see Fig. 10). It should be noted that the pattern of normative values is practically repeated by the pattern of values of individual priorities - the ranking of the values of universalism, independence, security, conformity and kindness coincides. However, it can also be noted that the value of tradition (respect, acceptance of customs and ideas) is more proclaimed than an individual priority, and the opposite situation occurs with the values of hedonism and stimulation - apparently, they are subject to a certain censorship at the level of normative values, then there are deliberately considered unimportant, but at the same time they are used as a practice at the level of individual priorities.

From the point of view of the author of the methodology (Schwartz), the block of values independence, stimulation and hedonism are an indicator of the individual's creative activity.

Correlation analysis showed that independence is interconnected with the 15th factor of moral choices "internal duty, responsibility to oneself" (p-0.01) and inversely interconnected with the 6th factor "role behavior - class ethics" (p-0.01). Stimulation (openness to novelty) is inversely related to the 10th factor "universal justice - one must reckon with others." Hedonism is inversely related to the 6th factor "role behavior - class ethics".

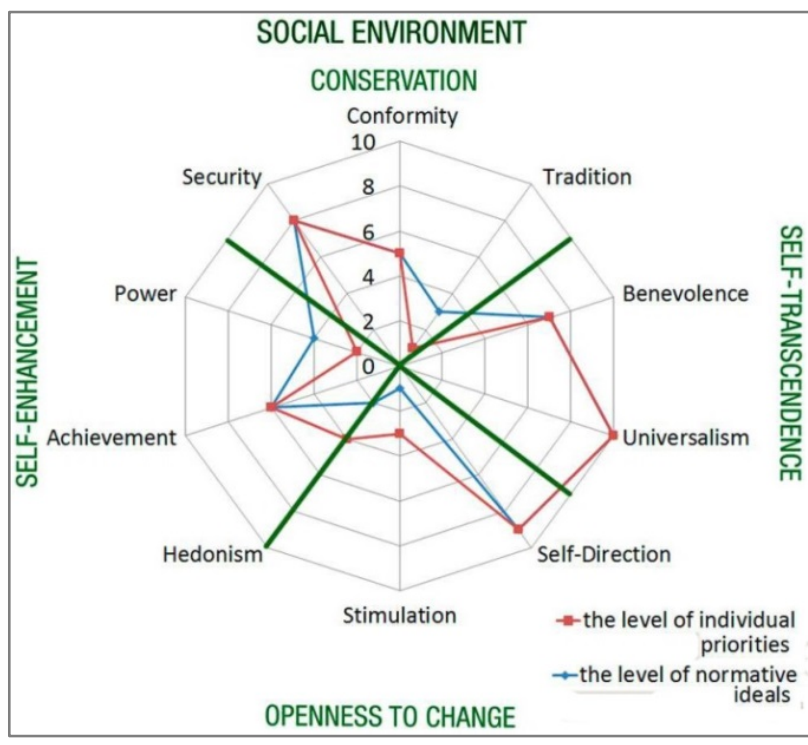

Fig. 10. Schwartz's circle of values for a sample of graduate students.

\subsection{Analysis and interpretation of the Längle's questionnaire for a sample of graduate students}

The methodology determines the quality of life of an individual associated with the feeling of life being filled with meaning. Existential fulfillment is interpreted as a feeling of accomplishment of the essential meaning, "real" life, the inclusion of one's potential in the dynamics of life.

The average value of the existential fulfillment scales (G-190.32) of the sample is in the middle zone below normal (see Fig. 11). The SD self-distancing subscale measures the stable ability for objective perception, the ability to free oneself from affects and prejudices. The mean SD (31.14) of the sample is below the norm according to the method (35.5) between selective perception and free attention for the perception of the world. The ST self-transcendence subscale measures the ability to feel values, to be guided not only by the goal, but also by the meaning of what is happening. The Freedom F subscale measures the ability to resolve. Responsibility subscale $\mathrm{V}$ measures the ability to act based on one's own values.

The analysis of indicators of existential fulfillment of the sample shows an active tendency towards an increase in internal openness, which gives a person the opportunity to go to the requests and offers of the outside world, sensitively correlating them with their own values. There is strength and emotional involvement, which is rather hampered by the constraint in the implementation of one's life. Inadequate norms and goals in the personality of an intellectual leader block openness and inclusion in life. Suppression of personality growth, leaving one's meaning for later leads to a reduction in freedom and responsibility, and can also lead to depression. 


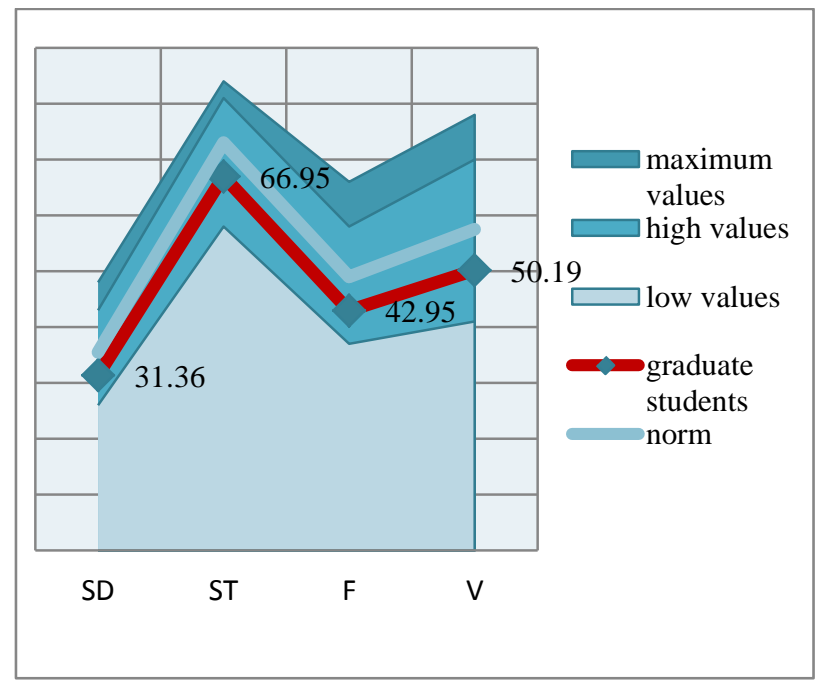

Fig. 11. Langle's Existence Scale for a sample of graduate students.

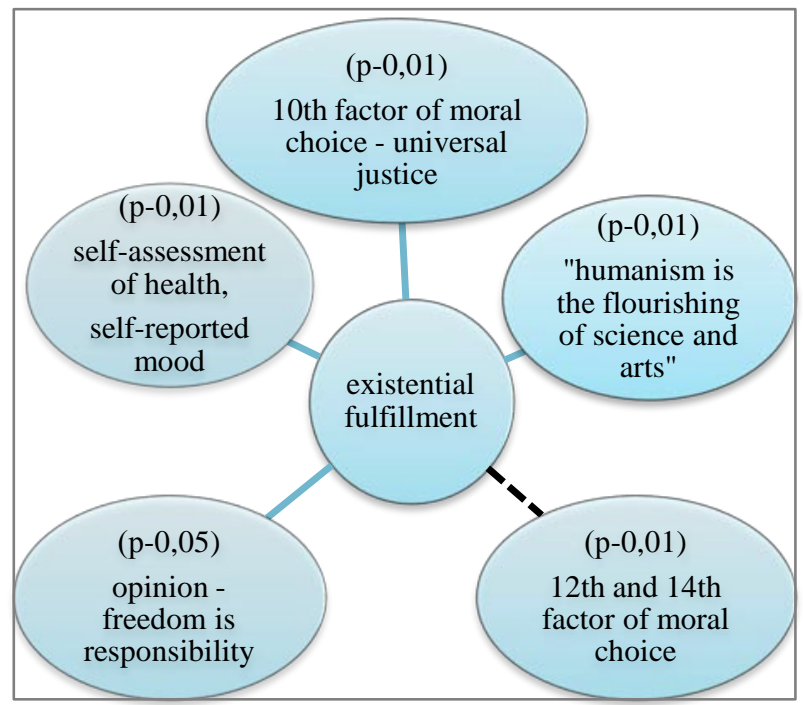

Fig. 12. Correlations with indicators of the scale of existential fulfillment $(\mathrm{G})$

The most significant interconnections of the parameters of existential fulfillment can be observed with self-esteem of health (see Fig. 12), with self-esteem of mood and vitality, the opinion that "freedom is first responsibility" and "humanism is the flourishing of science and arts". The parameters of existential fulfillment are inversely related to the factors of moral choice: pharisaism and religiosity (Dubov). And they have a direct relationship with the factor of moral choice - universal justice (it is necessary to consider the interests and rights of other people). The moral of universal justice in this interpretation can mean that an intellectual leader is a vector of development not only for himself, but also for others. Respect for others is a natural part of a social leader's personality.

\section{Conclusion}

In conclusion, we would like to note several features. The comprehension of the proposed questions by graduate students made it possible to highlight important interrelationships of personal positions. If a person's goal is the disclosure of his own abilities, potential, then it is realized through love for his own work, because in a humanistic society he is the main and responsible actor capable of self-realization. If creativity is considered the highest achievement of a person, then because it is a duty to oneself and humanism is then interpreted as the spiritual health of people. Those graduate students who view freedom as an opportunity for creative action expect society to support the initiative, which is certainly true from the point of view of building a humanistic society, but non-functional as a behavioral strategy.

An analysis of graduate students from the point of view of the values of a creative personality (independence, stimulation and hedonism) showed that the independence of thought and action is interconnected with the morality of internal duty, it is responsibility to oneself. The more independence and desire to enjoy life (hedonism) is manifested by a person, the less he or she is guided by class traditional ethical principles. I would like to note that pleasure in this case does not mean generally accepted, but precisely what satisfies the aesthetics of the leader. Openness to novelty and hedonism are reduced by postgraduate students' ideas about their own insignificance, lack of faith in their uniqueness, and a desire to meet the expectations of other people.

\section{References}

1. A. Meneghetti, From historical to eternal humanism, (Moscow: Antonio Meneghetti Scientific Foundation, 2014)

2. V.A. Dmitrieva, International symposium "Forming leadership for future development", UN, Geneva (Switzerland) (2019)

3. A. Menegetti. "An intellectual leader: what he can and should do" January 5, 2001 in New York (USA), in hall 1 of the UN Crystal Palace

4. A. Meneghetti, Youth and the Ethics of Life, (M .: Antonio Meneghetti Scientific Foundation, 2013)

5. M.V. Mikhaylova, M.A. Revina, EPJ Web of Conferences, 224, $06003 \quad$ (2019) https://doi.org/10.1051/epjconf/201922406003

6. W.F. Ryan, Ultimate Reality and Meaning, 36(1-2), 47-63 (2019)

https://doi.org/10.3138/uram.36.1-2.47

7. Sh. Schwartz et al, Psychology. Journal of the Higher School of Economics, 9(2), 43-70 (2012)

8. I.N. Mainina, A.Yu. Vasanov , Psychological Journal, 31(1), 87-99 (2010)

9. R. Kleszcz, Problems of Science, 53(1), 29-48 (2017)

10. I.G. Dubov, A.A. Khvostov, Questions of psychology, 5, 87-99 (2000) 